\title{
An Ambiguity Measure under EUUP and Its Application to a Portfolio Problem
}

\author{
Hideki Iwaki \\ Faculty of Business Administration, Kyoto Sangyo University, Kyoto, Japan \\ Email: iwaki@cc.kyoto-su.ac.jp
}

How to cite this paper: Iwaki, H. (2020) An Ambiguity Measure under EUUP and Its Application to a Portfolio Problem. Journal of Mathematical Finance, 10, 287-305. https://doi.org/10.4236/jmf.2020.102018

Received: March 7, 2020

Accepted: May 18, 2020

Published: May 21, 2020

Copyright (C) 2020 by author(s) and Scientific Research Publishing Inc. This work is licensed under the Creative Commons Attribution International License (CC BY 4.0).

http://creativecommons.org/licenses/by/4.0/

\begin{abstract}
This paper derives a measure that quantifies the degree of ambiguity under expected utility with uncertain probability (EUUP) by [1]. Here, ambiguity means a situation in which the first-order probabilities, i.e., the probabilities of the states of nature, are not given uniquely, but as random variables. Because EUUP can completely distinguish attitudes toward both risk and ambiguity from beliefs and risk from ambiguity, the derived ambiguity measure is independent of risk and attitudes toward both risk and ambiguity. We show that the degree of ambiguity can be measured by the variance of the first-order probabilities. Although [2] also derives an ambiguity measure based on the variance of the first-order probabilities, our measure is more flexible, and it discriminates between ambiguity in favorable outcomes and in unfavorable ones. Based on the measure, we also discuss effects of ambiguity on a problem of portfolio selection through comparative statics.
\end{abstract}

\section{Keywords}

Ambiguity Measurement, Knightian Uncertainty, Expected Utility with Uncertain Probability (EUUP), Portfolio Selection

\section{Introduction}

Uncertainty is used as an umbrella term for both risk and ambiguity. Risk is defined as a condition in which the event to be realized is a-priori unknown, but the odds of all possible events are perfectly known. Ambiguity refers to conditions in which not only the event to be realized is a-priori unknown, but the odds of the events are also either not uniquely assigned or are unknown. We are rarely able to avoid ambiguity in decision-making under uncertainty [3], and the search for models of decision making under ambiguity has been evolving toward the ultimate separations between tastes and beliefs, and between risk and ambi- 
guity [4] [5] [6] [7] [8] and [9]. However, as [1] says, some fundamental questions such as "How can the degree of ambiguity be measured? or What is the relationship between risk and ambiguity (preferences)?" remain unanswered. To answer these questions, [1] devises a new decision-making framework under uncertainty, called expected utility with uncertain probabilities (EUUP). EUUP can completely distinguish attitudes toward both risk and ambiguity from beliefs, and risk from ambiguity. It possesses a feature that permits ambiguity to be represented by uncertain multiple probability distributions over the states of nature, and permits preferences over ambiguity to be applied directly to these probability distributions.

This paper provides an applicable ambiguity measure under EUUP. As we show in the paper, the degree of ambiguity can be measured by the variance of probabilities. The ambiguity measure is independent of risk, simple, and intuitive. Although [2] also derives an ambiguity measure based on the variance of probabilities, he only considers the symmetric ambiguity case such that a decision maker (DM)'s ambiguity attitude is uniformly ambiguity averse or loving regardless of whether ambiguous outcomes are favorable or unfavorable for her/him. However, as pointed out by [10] and [11] based on their experiment, losses should be treated differently, with more ambiguity seeking than gain. Hence, instead of Izhakian's universal ambiguity measure, which is reference independent with respect to losses and gains, we derive an ambiguity measure that reflects differences between attitudes toward ambiguity for gains and ambiguity for losses. In many applications of ambiguity (asset markets, insurance, health, and so on) the gain-loss distinction is important, and ambiguity measures that assume reference-independent universal ambiguity aversion cannot accommodate this. Making ambiguity measures be reference dependent is the only generalization to accommodate this violation.

In the literature, most studies discuss universal ambiguity aversion without deriving an ambiguity measure that is independent of both risk and attitude toward risk; e.g. [9] [12]-[21]. Unlike those studies, our ambiguity measure is risk independent and can be applied to distinguish between loss and gain of outcomes.

In the financial markets, the future security returns are sometime represented mainly based on experts estimations due to lack of historical data [22]. ${ }^{1}$ This situation shows security returns must be considered ambiguous. Hence, in addition to deriving an ambiguity measure, this paper presents some comparative statistics about the standard portfolio problem in order to demonstrate the applicability of our ambiguity measure. We consider the impact of both ambiguity aversion and change in the extent of ambiguity on investment in uncertain assets whose returns create exposure to ambiguity.

This paper is organized as follows. We formally state our model in Section 2. Section 3 addresses the ordering of ambiguous acts and derives a measure of ambiguity. In Section 4, we present the application of the derived ambiguity ${ }^{1}[22]$ proposes a semivariance method for diversified portfolio selection, in which the security returns are given subjective to experts estimations and depicted as uncertain variables. 
measure to the portfolio problem and discuss the effects of ambiguity on financial investments based on comparative statics. The final section concludes.

\section{The Model}

In order to derive an ambiguity measure under EUUP, we set up the model to establish EUUP first. ${ }^{2}$ Let $\mathcal{S}$ be an infinite primary state space, and $\mathcal{E}$ be a $\sigma$ -algebra of subsets of $\mathcal{S}$. Let $X \subseteq \mathbb{R}$ be a convex set of consequences. We assume the interval $[0,1] \subset X$. Let $\kappa \in X$ be a reference point such that a consequence $x \in X$ is considered to be unfavorable if $x \leq \kappa$, and favorable if $\kappa<x$.

A bounded $\mathcal{E}$-measurable function $f: \mathcal{S} \rightarrow X$ is called a primary act. A simple primary act is a primary act that can be represented as a sequence of pairs, $f=\left(E_{1}: x_{1}, \cdots, E_{n}: x_{n}\right)$, where $\left(E_{1}, \cdots, E_{n}\right)$ is a generic partition of the state space $\mathcal{S} ; x_{j}$ is the consequence if event $E_{j}$ occurs; and the consequences $x_{1}, \cdots, x_{n}$ are listed in a non-decreasing order such that $x_{1} \leq \cdots \leq x_{k} \leq \kappa \leq x_{k+1} \leq \cdots \leq x_{n}$. We denote the set of all these simple measurable (Savage) acts by $\mathcal{F}_{0}$. A primary indicator act, $\delta_{E}=\left(E^{c}: 0, E: 1\right)$, assigns the outcome 1 if event $E \in \mathcal{E}$ occurs, and the outcome 0 otherwise.

Let $\mathcal{P}$ denote a set of all additive probability measures on the primary state space $\mathcal{S}$. A first-order probability measure $\mathrm{P} \in \mathcal{P}$ is then viewed as a state of nature in the secondary space $\mathcal{P}$. We assume the state space $\mathcal{P}$ is endowed with an algebra, $\Pi \subset 2^{\mathcal{P}}$, of subsets of $\mathcal{P}$. A bounded $\Pi$-measurable function $\stackrel{\Delta}{f}: \mathcal{P} \rightarrow X$, is called a secondary act. The set of all secondary acts is denoted by $\stackrel{\Delta}{F}$. A secondary act $\hat{\delta}_{E}: \mathcal{P} \rightarrow[0,1]$ is defined by $\hat{\delta}_{E}(\mathrm{P})=\mathrm{P}(E)$, corresponding to the expected outcome of a primary indicator act $\delta_{E} \in \mathcal{F}_{0}$. The subset of all such $\hat{\delta}_{E} \in \stackrel{\Delta}{F}$ is denoted by $\hat{\Delta}$.

Under the following Assumptions 1-4 $4^{3},[1]$ shows that the preference relation $\succsim^{1}$ over the set of primary acts $\mathcal{F}_{0}$ is given by a function $V: \mathcal{F}_{0} \rightarrow \mathbb{R}$, which is called expected utility with uncertain probabilities (EUUP), and defined by

$$
\begin{aligned}
V(f)= & \int_{z \leq 0}\left[\Upsilon_{-}^{-1}\left(\int_{\mathcal{P}} \Upsilon_{-}\left(\mathrm{P}\left\{s \in \mathcal{S} \mid U_{-}(f(s)) \geq z\right\}\right) \mathrm{d} \xi\right)-1\right] \mathrm{d} z \\
& +\int_{z>0} \Upsilon_{+}^{-1}\left(\int_{\mathcal{P}} \Upsilon_{+}\left(\mathrm{P}\left\{s \in \mathcal{S} \mid U_{+}(f(s)) \geq z\right\}\right) \mathrm{d} \xi\right) \mathrm{d} z, \forall f \in \mathcal{F}_{0} .
\end{aligned}
$$

Assumption 1. For every $c \in[0,1]$ there exists an event $E \in \mathcal{E}$ with the probability $\mathrm{P}(E)=c$.

${ }^{2}$ This setup is mostly adopted from [1].

${ }^{3}$ As to the validity of these assumptions, see [1].

${ }^{4}$ The original fully represented EUUP is given by

$$
\begin{aligned}
V(f)= & \int_{z \leq 0}\left[\Gamma_{-}\left(\Upsilon_{-}^{-1}\left(\int_{\mathcal{P}} \Upsilon_{-}\left(\mathrm{P}\left\{s \in \mathcal{S} \mid U_{-}(f(s)) \geq z\right\}\right) \mathrm{d} \xi\right)\right)-1\right] \mathrm{d} z \\
& +\int_{z>0} \Gamma_{+}\left(\Upsilon_{+}^{-1}\left(\int_{\mathcal{P}} \Upsilon_{+}\left(\mathrm{P}\left(\left\{s \in \mathcal{S} \mid U_{+}(f(s)) \geq z\right\}\right)\right) \mathrm{d} \xi\right)\right) \mathrm{d} z,
\end{aligned}
$$

where $\Gamma_{-}:[0,1] \rightarrow[0,1]$ and $\Gamma_{+}:[0,1] \rightarrow[0,1]$ are unique continuous strictly increasing functions, which form subjective distortions of perceived probabilities (probability weights) for loss and gain respectively. However, in order to hedge technical difficulties and obtain clear economic implications, it is assumed that the DM does not distort probability, i.e., $\Gamma_{-}$and $\Gamma_{+}$are the identity functions in the paper. 
Assumption 2. The preference relation $\succsim^{1}$ over the set of primary acts $\mathcal{F}_{0}$ satisfies the cumulative prospect theory axioms of [3].

Assumption 3. The preference relation $\succsim^{2}$ over the set of secondary acts $\stackrel{\Delta}{F}$ satisfies the extended Savage axioms of [23] and $x>y \Leftrightarrow x \succ^{2} y$, for all $x, y \in X$.

Assumption 4. Jointly, $\succsim^{1}$ and $\succsim^{2}$ satisfy the asymmetric certainty equivalent probabilistic consistency axiom (Axiom 2) of [1]. That is, let $\mathbf{1}_{F} \in \mathcal{F}_{0}$ be the unambiguous positive indicator act whose associated constant secondary act pays $c$, and let $-\mathbf{1}_{F} \in \mathcal{F}_{0}$ be the unambiguous negative indicator act whose associated constant secondary act pays $-d$, for some constants $c, d \in[0,1]$. For every event $E \in \mathcal{E}$,

$$
\begin{gathered}
\delta_{E} \sim_{1} \mathbf{1}_{F} \Leftrightarrow \hat{\delta}_{E} \sim_{2} c, \\
\text { and }-\delta_{E} \sim_{1}-\mathbf{1}_{F} \Leftrightarrow-\hat{\delta}_{E} \sim_{2}-d .
\end{gathered}
$$

We note that because $\hat{\delta}_{E} \in \hat{\Delta}$ is identified with the first-order probability $\{\mathrm{P}(E)\}_{\mathrm{P} \in \mathcal{P}}$ of event $E$, the preference relation $\succsim^{2}$ over $\hat{\Delta}$ can be considered as a preference over the first-order probabilities. Assumption 3 about $\succsim^{2}$ implies a unique second-order countably additive probability measure $\xi$ on $\Pi$ that assigns each subset $A \in \Pi$ of first-order probability measures in $\mathcal{P}$ with a probability $\xi(A)$.

In (1), the function $V$, representing the DM's preferences, takes the form of a reference-dependent Choquet integration to unfavorable and favorable outcomes relative to the reference point $\kappa$. In the sequel, we assume $\kappa$ to be 0 without loss of generality. $V$ distinguishes beliefs from attitudes toward risk and ambiguity, and risk from ambiguity, as follows. The first-order belief, which captures risk, is given by the uncertain finitely additive probability measure $\mathrm{P} \in \mathcal{P}$. The second-order belief, which captures ambiguity, is given by a unique non-atomic countably additive probability measure $\xi$ over the set $\mathcal{P}$ of the first-order probability measures over $\mathcal{S}$. Attitudes toward risk are given by the utility functions $U_{-}: X_{-}=\{x \in X \mid x \leq 0\} \rightarrow \mathbb{R}$ and $U_{+}: X_{+}=\{x \in X \mid x>0\} \rightarrow \mathbb{R}$, which are continuous strictly increasing bounded functions and unique up to a positive scaling, normalized such that $U_{+}(\kappa=0)=U_{-}(\kappa=0)=0$. Here, $U_{-}$represents the DM's utility for loss, i.e., negative outcomes, and $U_{+}$represents the DM's utility for gain, i.e., positive outcomes. Attitudes toward ambiguity are given by continuous non-constant bounded functions $\Upsilon_{-}:[0,1] \rightarrow \mathbb{R}$ and $\Upsilon_{+}:[0,1] \rightarrow \mathbb{R}$, referred to as an outlook function for loss and outlook function for gain, respectively. $\Upsilon_{-}$and $\Upsilon_{+}$are unique up to a positive linear transformation.

For each $f \in \mathcal{F}_{0}$, without loss of generality, we can denote $f=\sum_{i=1}^{n} x_{i} \delta_{E_{i}}$, where $\left\{x_{i} \in \mathbb{R}: i=1, \cdots, n\right\}$ is a strict increasing sequence of real numbers and $\left\{E_{i} \subset S: i=1, \cdots, n\right\}$ is a partition of $\mathcal{S} .^{5}$

For each $f \in \mathcal{F}_{0}$, we also denote $\mathrm{P}_{f}\left(x_{i}\right)=\mathrm{P}\left(\left\{s \in \mathcal{S} \mid f(s)=x_{i}\right\}\right)$. Then, we

${ }^{5}$ That is, $\sum_{i=1}^{n} E_{i}=\mathcal{S}$, where $\sum_{i=1}^{n} E_{i}$ denotes $\bigcup_{i=1}^{n} E_{i}$, for mutually disjoint events $E_{i}, E_{j} \subset \mathcal{S}$, $i, j=1, \cdots, n$, such that $E_{i} \cap E_{j}=\varnothing$. 
can easily show that we can rewrite (1) more explicitly as

$$
\begin{aligned}
V(f)= & \sum_{i=k+1}^{n} U_{+}\left(x_{i}\right)\left(\Upsilon_{+}^{-1}\left(\mathrm{E}\left[\Upsilon_{+}\left(\sum_{j=i}^{n} \mathrm{P}_{f}\left(x_{j}\right)\right)\right]\right)-\Upsilon_{+}^{-1}\left(\mathrm{E}\left[\Upsilon_{+}\left(\sum_{j=i+1}^{n} \mathrm{P}_{f}\left(x_{j}\right)\right)\right]\right)\right) \\
& +\sum_{i=1}^{k} U_{-}\left(x_{i}\right)\left(\hat{\Upsilon}_{-}^{-1}\left(\mathrm{E}\left[\hat{\Upsilon}_{-}\left(\sum_{j=1}^{i} \mathrm{P}_{f}\left(x_{j}\right)\right)\right]\right)-\hat{\Upsilon}_{-}^{-1}\left(\mathrm{E}\left[\hat{\Upsilon}_{-}\left(\sum_{j=1}^{i-1} \mathrm{P}_{f}\left(x_{j}\right)\right)\right]\right)\right)
\end{aligned}
$$

where $\hat{\Upsilon}_{-}$is the dual of $\Upsilon_{-}$defined by $\hat{\Upsilon}_{-}^{-1}\left(\mathrm{E}\left[\hat{\Upsilon}_{-}(\mathrm{P}(E))\right]\right)=1-\Upsilon_{-}^{-1}\left(\mathrm{E}\left[\Upsilon_{-}\left(\mathrm{P}\left(E^{c}\right)\right)\right]\right)$ for event $E$ and its complementary event $E^{c}$, and where

$$
\mathrm{E}\left[\hat{\Upsilon}_{-}(\cdot)\right]=\int_{\mathcal{P}} \hat{\Upsilon}_{-}(\cdot) \mathrm{d} \xi \text {, and } \mathrm{E}\left[\Upsilon_{+}(\cdot)\right]=\int_{\mathcal{P}} \Upsilon_{+}(\cdot) \mathrm{d} \xi
$$

that is, E denotes expectation under the second-order probability measure $\xi$. From (2), if $\Upsilon_{-}$and $\Upsilon_{+}$are the identity functions or the DM is ambiguity neutral, and $U_{-}=U_{+}=U$,

$$
V(f)=\sum_{i=1}^{n} U\left(x_{i}\right) \mathrm{E}\left[\mathrm{P}_{f}\left(x_{i}\right)\right] .
$$

Hence, in this case, it is considered as the expected utility (EU) where the probability of the outcome $x_{i}$ occurring is $\mathrm{E}\left[\mathrm{P}_{f}\left(x_{i}\right)\right]$.

We derive a measure of ambiguity based on (2). In parallel with expressing the risk by variance under EU, our main idea is as follows. Because ambiguity is represented by the assumption that the first-order probability is a random variable, we try to quantify the degree of ambiguity by variance of the first-order probability under the second-order probability measure. However, because the functional representation $V$ is somewhat complicated, it should be simplified. We make a simplification by applying a quadratic approximation to the DM's perceived probabilities or capacities $\Upsilon_{+}^{-1}\left(E\left[\Upsilon_{+}(\cdot)\right]\right)$ and $\hat{\Upsilon}_{-}^{-1}\left(E\left[\hat{\Upsilon}_{-}(\cdot)\right]\right)$. By using this approximation, we can represent the DM's perceived probabilities by a function of means and variances of the first-order probabilities under the second-order probabilities. The result is given by the following theorem.

Theorem 1. Suppose the outlook functions $\Upsilon_{+}$and $\Upsilon_{-}$are twice continuously differentiable. Then, the following equation holds.

$$
\begin{aligned}
V(f)= & \sum_{i=k+1}^{n} U_{+}\left(x_{i}\right)\left(\mathrm{E}\left[\mathrm{P}_{f}\left(x_{i}\right)\right]+\frac{1}{2} \frac{\Upsilon_{+}^{\prime \prime}\left(\sum_{j=i}^{n} \mathrm{E}\left[\mathrm{P}_{f}\left(x_{j}\right)\right]\right)}{\Upsilon_{+}^{\prime}\left(\sum_{j=i}^{n} \mathrm{E}\left[\mathrm{P}_{f}\left(x_{j}\right)\right]\right)} \operatorname{Var}\left[\sum_{j=i}^{n} \mathrm{P}_{f}\left(x_{j}\right)\right]\right. \\
& \left.-\frac{1}{2} \frac{\Upsilon_{+}^{\prime \prime}\left(\sum_{j=i+1}^{n} \mathrm{E}\left[\mathrm{P}_{f}\left(x_{j}\right)\right]\right)}{\Upsilon_{+}^{\prime}\left(\sum_{j=i+1}^{n} \mathrm{E}\left[\mathrm{P}_{f}\left(x_{j}\right)\right]\right)} \operatorname{Var}\left[\sum_{j=i+1}^{n} \mathrm{P}_{f}\left(x_{j}\right)\right]\right) \\
& +\sum_{i=1}^{k} U_{-}\left(x_{i}\right)\left(\mathrm{E}\left[\mathrm{P}_{f}\left(x_{i}\right)\right]+\frac{1}{2} \frac{\hat{\Upsilon}_{-}^{\prime \prime}\left(\sum_{j=1}^{i} \mathrm{E}\left[\mathrm{P}_{f}\left(x_{j}\right)\right]\right)}{\hat{\Upsilon}_{-}^{\prime}\left(\sum_{j=1}^{i} \mathrm{E}\left[\mathrm{P}_{f}\left(x_{j}\right)\right]\right)} \operatorname{Var}\left[\sum_{j=1}^{i} \mathrm{P}_{f}\left(x_{j}\right)\right]\right. \\
& -\frac{1}{2} \frac{\hat{\Upsilon}_{-}^{\prime \prime}\left(\sum_{j=1}^{i-1} \mathrm{E}\left[\mathrm{P}_{f}\left(x_{j}\right)\right]\right)}{\hat{\Upsilon}_{-}^{\prime}\left(\sum_{j=1}^{i-1} \mathrm{E}\left[\mathrm{P}_{f}\left(x_{j}\right)\right]\right)} \operatorname{Var}\left[\sum_{j=1}^{i-1} \mathrm{P}_{f}\left(x_{j}\right)\right]+o\left(\operatorname{Var}\left[\sum_{i=1}^{n} \mathrm{P}_{f}\left(x_{i}\right)\right]\right)
\end{aligned}
$$


where $\operatorname{Var}\left[\sum_{i=1}^{n} \mathrm{P}_{f}\left(x_{i}\right)\right]$ is the variance of $\sum_{i=1}^{n} \mathrm{P}_{f}\left(x_{i}\right)$ under the secondorder probability measure $\xi$ and $o(\cdot)$ is the small order.

Proof. For each $j=k+1, \cdots, n$, expand $\Upsilon_{+}(\cdot)$ around $\sum_{i=j}^{n} \mathrm{E}\left[\mathrm{P}_{f}\left(x_{i}\right)\right]$, we have

$$
\begin{aligned}
& \Upsilon_{+}\left(\sum_{i=j}^{n} \mathrm{P}_{f}\left(x_{i}\right)\right) \\
& =\Upsilon_{+}\left(\sum_{i=j}^{n} \mathrm{E}\left[\mathrm{P}_{f}\left(x_{i}\right)\right]\right)+\Upsilon_{+}^{\prime}\left(\sum_{i=j}^{n} \mathrm{E}\left[\mathrm{P}_{f}\left(x_{i}\right)\right]\right) \sum_{i=j}^{n}\left(\mathrm{P}_{f}\left(x_{i}\right)-\mathrm{E}\left[\mathrm{P}_{f}\left(x_{i}\right)\right]\right) \\
& +\frac{1}{2} \Upsilon_{+}^{\prime \prime}\left(\sum_{i=j}^{n} \mathrm{E}\left[\mathrm{P}_{f}\left(x_{i}\right)\right]\right)\left(\sum_{i=j}^{n}\left(\mathrm{P}_{f}\left(x_{i}\right)-\mathrm{E}\left[\mathrm{P}_{f}\left(x_{i}\right)\right]\right)\right)^{2} \\
& +o\left(\sum_{i=j}^{n}\left(\mathrm{P}_{f}\left(x_{i}\right)-\mathrm{E}\left[\mathrm{P}_{f}\left(x_{i}\right)\right]\right)\right)^{2} .
\end{aligned}
$$

Applying the expectation $\mathrm{E}[\cdot]$ to the above equation, we have

$$
\begin{aligned}
& \mathrm{E}\left[\Upsilon_{+}\left(\sum_{i=j}^{n} \mathrm{P}_{f}\left(x_{i}\right)\right]\right] \\
& =\Upsilon_{+}\left(\sum_{i=j}^{n} \mathrm{E}\left[\mathrm{P}_{f}\left(x_{i}\right)\right]\right)+\frac{1}{2} \Upsilon_{+}^{\prime \prime}\left(\sum_{i=j}^{n} \mathrm{E}\left[\mathrm{P}_{f}\left(x_{i}\right)\right]\right) \operatorname{Var}\left[\sum_{i=j}^{n} \mathrm{P}_{f}\left(x_{i}\right)\right] \\
& +o\left(\operatorname{Var}\left[\sum_{i=j}^{n} \mathrm{P}_{f}\left(x_{i}\right)\right]\right) .
\end{aligned}
$$

From this, we have

$$
\begin{aligned}
& \Upsilon_{+}^{-1}\left(\mathrm{E}\left[\Upsilon_{+}\left(\sum_{i=j}^{n} \mathrm{P}_{f}\left(x_{i}\right)\right)\right]\right) \\
& =\Upsilon_{+}^{-1}\left(\Upsilon_{+}\left(\sum_{i=j}^{n} \mathrm{E}\left[\mathrm{P}_{f}\left(x_{i}\right)\right]\right)+\frac{1}{2} \Upsilon_{+}^{\prime \prime}\left(\sum_{i=j}^{n} \mathrm{E}\left[\mathrm{P}_{f}\left(x_{i}\right)\right]\right) \operatorname{Var}\left[\sum_{i=j}^{n} \mathrm{P}_{f}\left(x_{i}\right)\right]\right) \\
& +o\left(\operatorname{Var}\left[\sum_{i=j}^{n} \mathrm{P}_{f}\left(x_{i}\right)\right]\right) .
\end{aligned}
$$

Furthermore, expanding $\Upsilon_{+}^{-1}(\cdot)$ around $\Upsilon_{+}\left(\sum_{i=j}^{n} \mathrm{E}\left[\mathrm{P}_{f}\left(x_{i}\right)\right]\right)$, we have

$$
\begin{aligned}
& \Upsilon_{+}^{-1}\left(\Upsilon_{+}\left(\sum_{i=j}^{n} \mathrm{E}\left[\mathrm{P}_{f}\left(x_{i}\right)\right]\right)+\frac{1}{2} \Upsilon_{+}^{\prime \prime}\left(\sum_{i=j}^{n} \mathrm{E}\left[\mathrm{P}_{f}\left(x_{i}\right)\right]\right) \operatorname{Var}\left[\sum_{i=j}^{n} \mathrm{P}\left(x_{i}\right)\right]\right) \\
& =\Upsilon_{+}^{-1}\left(\Upsilon_{+}\left(\sum_{i=j}^{n} \mathrm{E}\left[\mathrm{P}_{f}\left(x_{i}\right)\right]\right)\right)+\left(\Upsilon_{+}^{-1}\right)^{\prime}\left(\Upsilon_{+}\left(\sum_{i=j}^{n} \mathrm{E}\left[\mathrm{P}_{f}\left(x_{i}\right)\right]\right)\right) \\
& \times \frac{1}{2} \Upsilon_{+}^{\prime \prime}\left(\sum_{i=j}^{n} \mathrm{E}\left[\mathrm{P}_{f}\left(x_{i}\right)\right]\right) \operatorname{Var}\left[\sum_{i=j}^{n} \mathrm{P}_{f}\left(x_{i}\right)\right]+o\left(\operatorname{Var}\left[\sum_{i=j}^{n} \mathrm{P}_{f}\left(x_{i}\right)\right]\right) \\
& =\sum_{i=j}^{n} \mathrm{E}\left[\mathrm{P}_{f}\left(x_{i}\right)\right]+\frac{1}{2} \frac{\Upsilon_{+}^{\prime \prime}\left(\sum_{i=j}^{n} \mathrm{E}\left[\mathrm{P}_{f}\left(x_{i}\right)\right]\right)}{\Upsilon_{+}^{\prime}\left(\sum_{i=j}^{n} \mathrm{E}\left[\mathrm{P}_{f}\left(x_{i}\right)\right]\right)} \operatorname{Var}\left[\sum_{i=j}^{n} \mathrm{P}_{f}\left(x_{i}\right)\right] \\
& +o\left(\operatorname{Var}\left[\sum_{i=j}^{n} \mathrm{P}_{f}\left(x_{i}\right)\right]\right) .
\end{aligned}
$$


Hence, from (4), we have

$$
\begin{aligned}
& \Upsilon_{+}^{-1}\left(\mathrm{E}\left[\Upsilon_{+}\left(\sum_{i=j}^{n} \mathrm{P}_{f}\left(x_{i}\right)\right)\right]\right) \\
& =\sum_{i=j}^{n} \mathrm{E}\left[\mathrm{P}_{f}\left(x_{i}\right)\right]+\frac{1}{2} \frac{\Upsilon_{+}^{\prime \prime}\left(\sum_{i=j}^{n} \mathrm{E}\left[\mathrm{P}_{f}\left(x_{i}\right)\right]\right)}{\Upsilon_{+}^{\prime}\left(\sum_{i=j}^{n} \mathrm{E}\left[\mathrm{P}_{f}\left(x_{i}\right)\right]\right)} \operatorname{Var}\left[\sum_{i=j}^{n} \mathrm{P}_{f}\left(x_{i}\right)\right] \\
& +o\left(\operatorname{Var}\left[\sum_{i=j}^{n} \mathrm{P}_{f}\left(x_{i}\right)\right]\right)
\end{aligned}
$$

Similarly, we have

$$
\begin{aligned}
& \hat{\Upsilon}_{-}^{-1}\left(\mathrm{E}\left[\hat{\Upsilon}_{-}\left(\sum_{i=1}^{j} \mathrm{P}_{f}\left(x_{i}\right)\right)\right]\right) \\
& =\sum_{i=1}^{j} \mathrm{E}\left[\mathrm{P}_{f}\left(x_{i}\right)\right]+\frac{1}{2} \frac{\hat{\Upsilon}_{-}^{\prime \prime}\left(\sum_{i=1}^{j} \mathrm{E}\left[\mathrm{P}_{f}\left(x_{i}\right)\right]\right)}{\hat{\Upsilon}_{-}^{\prime}\left(\sum_{i=1}^{j} \mathrm{E}\left[\mathrm{P}_{f}\left(x_{i}\right)\right]\right)} \operatorname{Var}\left[\sum_{i=1}^{j} \mathrm{P}_{f}\left(x_{i}\right)\right] \\
& +o\left(\operatorname{Var}\left[\sum_{i=1}^{j} \mathrm{P}_{f}\left(x_{i}\right)\right]\right), \quad j=1, \cdots, k .
\end{aligned}
$$

Substituting (5) and (6) into (2), we obtain the results.

\section{Ordering Ambiguous Acts and an Ambiguity Measure}

We derive an ambiguity measure based on ordering ambiguous acts by DMs. Prior to deriving the measure, we define the DM's ambiguity attitudes. We note that from Assumptions 1 and 4, for each event $E \in \mathcal{E}$ there is an unambiguous event $E^{*} \in \mathcal{E}$ such that

$$
\mathrm{P}\left(E^{*}\right)=\mathrm{E}[\mathrm{P}(E)] \text { for all } \mathrm{P} \in \mathcal{P} .
$$

\section{Definition 1.}

1. A DM is ambiguity averse (neutral, loving) for gain if, for each event $E \in \mathcal{E}$ and event $E^{*} \in \mathcal{E}$ satisfying (7),

$$
\delta_{E^{*}} \succsim^{1} \delta_{E} \quad\left(\delta_{E^{*}} \sim^{1} \delta_{E}, \delta_{E^{*}} \precsim^{1} \delta_{E}\right) .
$$

2. A DM is ambiguity averse (neutral, loving) for loss if, for each event $E \in \mathcal{E}$ and event $E^{*} \in \mathcal{E}$ satisfying (7),

$$
-\delta_{E^{*}} \succsim^{1}-\delta_{E} \quad\left(-\delta_{E^{*}} \sim^{1}-\delta_{E},-\delta_{E^{*}} \precsim^{1}-\delta_{E}\right) .
$$

From Axiom 1 (certainty equivalent probabilistic constancy) of [1], "ambiguity averse (neutral, loving) for gain" and "ambiguity averse (neutral, loving) for loss" in Definition 1 are equivalent to "ambiguity averse (neutral, loving) as regards a favorable event" and "ambiguity averse (neutral, loving) as regards an unfavorable event" in Definition 5 of [1], respectively.

Next, we characterize a DM's ambiguity attitude toward gain and against loss by the shape of outlook function $\Upsilon_{+}$and the dual $\hat{\Upsilon}_{-}$of $\Upsilon_{-}$respectively.

Lemma 1. 
1. A DM is ambiguity averse (neutral, loving) for gain if and only if $\Upsilon_{+}$is concave (linear, convex).

2. A DM is ambiguity averse (neutral, loving) for loss if and only if $\hat{\Upsilon}_{-}$is convex (linear, concave).

Proof. We only show the case where a DM is ambiguity loving for loss because the proofs of other cases are quite similar. From Definition 1, we have only to show

$$
-\delta_{E} \succsim^{1}-\delta_{E^{*}} \quad \forall E \in \mathcal{E} \Leftrightarrow \hat{\Upsilon}_{-} \text {is concave. }
$$

Because

$$
\begin{aligned}
& \mathrm{P}\left(\left\{s \in \mathcal{S}: U_{-}\left(-\delta_{E}(s)\right)>t\right\}\right) \\
& = \begin{cases}\mathrm{P}(\Omega)=1 & \text { if } t<U_{-}(-1) \\
\mathrm{P}\left(E^{c}\right) & \text { if } U_{-}(-1) \leq t<U_{-}(0) \equiv 0 \\
\mathrm{P}(\varnothing)=0 & \text { if } t \geq U_{-}(0) \equiv 0,\end{cases}
\end{aligned}
$$

we have

$$
\begin{aligned}
V\left(-\delta_{E}\right)= & \int_{t \leq 0}\left(\Upsilon_{-}^{-1}\left(\int_{\mathrm{P} \in \mathcal{P}} \Upsilon_{-}\left(\mathrm{P}\left(\left\{s \in \mathcal{S}: U_{-}\left(-\delta_{E}(s)\right)>t\right\}\right)\right) \mathrm{d} \xi\right)-1\right) \mathrm{d} t \\
& +\int_{t>0} \Upsilon_{+}^{-1}\left(\int_{\mathrm{P} \in \mathcal{P}} \Upsilon_{+}\left(\mathrm{P}\left(\left\{s \in \mathcal{S}: U_{+}\left(-\delta_{E}(s)\right)>t\right\}\right)\right) \mathrm{d} \xi\right) \mathrm{d} t \\
= & \int_{U_{-}(-1)}^{0}\left(\Upsilon_{-}^{-1}\left(\int_{\mathrm{P} \in \mathcal{P}} \Upsilon_{-}\left(\mathrm{P}\left(E^{c}\right)\right) \mathrm{d} \xi\right)-1\right) \mathrm{d} t \\
= & -\left(\Upsilon_{-}^{-1}\left(\mathrm{E}\left[\Upsilon_{-}\left(\mathrm{P}\left(E^{c}\right)\right)\right]\right)-1\right) U_{-}(-1) \\
= & \hat{\Upsilon}_{-}^{-1}\left(\mathrm{E}\left[\hat{\Upsilon}_{-}(\mathrm{P}(E))\right]\right) U_{-}(-1) .
\end{aligned}
$$

On the other hand,

$$
\begin{aligned}
V\left(-\delta_{E^{*}}\right)= & \int_{t \leq 0}\left(\Upsilon_{-}^{-1}\left(\int_{\mathrm{P} \in \mathcal{P}} \Upsilon_{-}\left(\mathrm{P}\left(\left\{s \in \mathcal{S}: U_{-}\left(-\delta_{E^{*}}(s)\right)>t\right\}\right)\right) \mathrm{d} \xi\right)-1\right) \mathrm{d} t \\
& +\int_{t>0} \Upsilon_{-}^{-1}\left(\int_{\mathrm{P} \in \mathcal{P}} \Upsilon_{-}\left(\mathrm{P}\left(\left\{s \in \mathcal{S}: U_{+}\left(-\delta_{E^{*}}(s)\right)>t\right\}\right)\right) \mathrm{d} \xi\right) \mathrm{d} t \\
= & \int_{U_{-}(-1)}^{0}\left(\Upsilon_{-1}\left(\int_{\mathrm{P} \in \mathcal{P}} \Upsilon_{-}\left(\mathrm{P}\left(E^{* c}\right)\right) \mathrm{d} \xi\right)-1\right) \mathrm{d} t \\
= & -\left(\Upsilon_{-}^{-1}\left(\Upsilon_{-}\left(\mathrm{E}\left[\mathrm{P}\left(E^{c}\right)\right]\right)\right)-1\right) U_{-}(-1) \\
= & \hat{\Upsilon}_{-}^{-1}\left(\hat{\Upsilon}_{-}(\mathrm{E}[\mathrm{P}(E)])\right) U_{-}(-1),
\end{aligned}
$$

where we have used the fact that $\mathrm{P}\left(E^{*^{c}}\right)=\mathrm{E}\left[\mathrm{P}\left(E^{c}\right)\right]$ in the third equation. Here, noting $\hat{\Upsilon}_{-}^{-1}$ is increasing and $U_{-}(-1)<0$, we obtain the result from Jensen's inequality.

[1] defines "more ambiguous acts" without considering the difference in ambiguity attitude toward favorable outcomes and unfavorable results. Based on Definition 1, we expand the definition of "more ambiguous acts" by [1] and define the ordering of acts by the degree of ambiguity as follows.

Definition 2. For every $f \in \mathcal{F}$, let $f^{+}$and $f^{-}$denote the plus part and 
the minus part of $f$ respectively, i.e., $f^{+}=\max \{f, 0\}$ and $f^{-}=\min \{f, 0\}$. Let $f, g \in \mathcal{F}_{0}$ be two primary acts under which the expected probabilities of each consequence $x \in X$ are identical, i.e., $\mathrm{E}\left[\mathrm{P}_{f}(x)\right]=\mathrm{E}\left[\mathrm{P}_{g}(x)\right]$, for every $x \in X$.

1. Act $g$ is more (less) ambiguous than $f$ for gain if and only if

$$
f^{+} \succsim^{1} g^{+} \quad\left(f^{+} \precsim^{1} g^{+}\right)
$$

by any DM who is ambiguity averse for gain.

2. Act $g$ is less (more) ambiguous than $f$ for loss if and only if

$$
f^{-} \succsim^{1} g^{-} \quad\left(f^{-} \precsim^{1} g^{-}\right)
$$

by any DM who is ambiguity loving for loss.

In Definition 2, we make an assumption that $\mathrm{E}\left[\mathrm{P}_{f}(x)\right]=\mathrm{E}\left[\mathrm{P}_{g}(x)\right]$ for every $x \in X$ to compare two primary acts $f, g \in \mathcal{F}_{0}$. This assumption is analogous that of [24] in that risk measurement is considered based on the order of lotteries with the same expected outcome.

The following theorem asserts that the degree of ambiguity associated with a primary act can be measured by "the variance of the first-order probabilities".

Theorem 2. Suppose that the condition of Theorem 1 is satisfied, a DM is ambiguity averse (loving) for gain and ambiguity loving (averse) for loss, and both of her outlook function for gain $\Upsilon_{+}$and the dual $\hat{\Upsilon}_{-}$of it for loss are of the increasing absolute ambiguity aversion (IAAA) type, i.e., $-\frac{\Upsilon_{+}^{\prime \prime}}{\Upsilon_{+}^{\prime}}$ and $-\frac{\hat{\Upsilon}_{-}^{\prime \prime}}{\hat{\Upsilon}_{-}^{\prime}}$ are increasing.

For every act $f, g \in \mathcal{F}_{0}$ under which the expected probabilities of each consequence $x \in X$ are identical and the probabilities of each $x \in X$ are mutually independent, if the variances satisfies

$$
\begin{gathered}
\operatorname{Var}\left[\mathrm{P}_{f}(x)\right] \leq(\geq) \operatorname{Var}\left[\mathrm{P}_{g}(x)\right], \quad \text { for all positive } x \in X, \\
\text { and } \operatorname{Var}\left[\mathrm{P}_{f}(x)\right] \geq(\leq) \operatorname{Var}\left[\mathrm{P}_{g}(x)\right], \quad \text { for all non-positive } x \in X .
\end{gathered}
$$

Then, ignoring the small order

$$
o\left(\sum_{j=1}^{n}\left(\operatorname{Var}\left[\mathrm{P}_{f}\left(x_{j}\right)\right]+\operatorname{Var}\left[\mathrm{P}_{g}\left(x_{j}\right)\right]\right)\right),
$$

the DM prefers $f$ to $g$, that is,

$$
f \succsim^{1} g
$$

Furthermore, if both $\Upsilon_{+}$and $\hat{\Upsilon}_{-}$are of the constant absolute ambiguity aversion (CAAA) type, i.e., $-\frac{\Upsilon_{+}^{\prime \prime}}{\Upsilon_{+}^{\prime}}$ and $-\frac{\hat{\Upsilon}_{-}^{\prime \prime}}{\hat{\Upsilon}_{-}^{\prime}}$ are constants, then (8) is the necessary and sufficient condition for (10).

Proof. From Theorem 1, 


$$
\begin{aligned}
& 2(V(f)-V(g)) \\
& =\sum_{i=k+1}^{n}\left(\frac{\Upsilon_{+}^{\prime \prime}\left(\sum_{j=i}^{n} \mathrm{E}\left[\mathrm{P}_{f}\left(x_{j}\right)\right]\right)}{\Upsilon_{+}^{\prime}\left(\sum_{j=i}^{n} \mathrm{E}\left[\mathrm{P}_{f}\left(x_{j}\right)\right]\right)}\left(\operatorname{Var}\left[\sum_{j=i}^{n} \mathrm{P}_{f}\left(x_{j}\right)\right]-\operatorname{Var}\left[\sum_{j=i}^{n} \mathrm{P}_{g}\left(x_{j}\right)\right]\right)\right. \\
& \left.-\frac{\Upsilon_{+}^{\prime \prime}\left(\sum_{j=i+1}^{n} \mathrm{E}\left[\mathrm{P}_{f}\left(x_{j}\right)\right]\right)}{\Upsilon_{+}^{\prime}\left(\sum_{j=i+1}^{n} \mathrm{E}\left[\mathrm{P}_{f}\left(x_{j}\right)\right]\right)}\left(\operatorname{Var}\left[\sum_{j=i+1}^{n} \mathrm{P}_{f}\left(x_{j}\right)\right]-\operatorname{Var}\left[\sum_{j=i+1}^{n} \mathrm{P}_{g}\left(x_{j}\right)\right]\right)\right) U_{+}\left(x_{i}\right) \\
& +\sum_{i=1}^{k}\left(\frac{\hat{\Upsilon}_{-}^{\prime \prime}\left(\sum_{j=1}^{i} \mathrm{E}\left[\mathrm{P}_{f}\left(x_{j}\right)\right]\right)}{\hat{\Upsilon}_{-}^{\prime}\left(\sum_{j=1}^{i} \mathrm{E}\left[\mathrm{P}_{f}\left(x_{j}\right)\right]\right)}\left(\operatorname{Var}\left[\sum_{j=1}^{i} \mathrm{P}_{f}\left(x_{j}\right)\right]-\operatorname{Var}\left[\sum_{j=1}^{i} \mathrm{P}_{g}\left(x_{j}\right)\right]\right)\right. \\
& \left.-\frac{\hat{\Upsilon}_{-}^{\prime \prime}\left(\sum_{j=1}^{i-1} \mathrm{E}\left[\mathrm{P}_{f}\left(x_{j}\right)\right]\right)}{\hat{\Upsilon}_{-}^{\prime}\left(\sum_{j=1}^{i-1} \mathrm{E}\left[\mathrm{P}_{f}\left(x_{j}\right)\right]\right)}\left(\operatorname{Var}\left[\sum_{j=1}^{i-1} \mathrm{P}_{f}\left(x_{j}\right)\right]-\operatorname{Var}\left[\sum_{j=1}^{i-1} \mathrm{P}_{g}\left(x_{j}\right)\right]\right)\right) U_{-}\left(x_{i}\right) \\
& +o\left(\operatorname{Var}\left[\sum_{i=1}^{n} \mathrm{P}_{f}\left(x_{i}\right)\right]\right)+o\left(\operatorname{Var}\left[\sum_{i=1}^{n} \mathrm{P}_{g}\left(x_{i}\right)\right]\right) \\
& =\sum_{i=k+1}^{n}\left\{\frac { \Upsilon _ { + } ^ { \prime \prime } ( \sum _ { j = i } ^ { n } \mathrm { E } [ \mathrm { P } _ { f } ( x _ { j } ) ] ) } { \Upsilon _ { + } ^ { \prime } ( \sum _ { j = i } ^ { n } \mathrm { E } [ \mathrm { P } _ { f } ( x _ { j } ) ] ) } \left(\operatorname{Var}\left[\sum_{j=i}^{n} \mathrm{P}_{f}\left(x_{j}\right)\right]-\operatorname{Var}\left[\sum_{j=i+1}^{n} \mathrm{P}_{f}\left(x_{j}\right)\right]\right.\right. \\
& \left.-\left(\operatorname{Var}\left[\sum_{j=i}^{n} \mathrm{P}_{g}\left(x_{j}\right)\right]-\operatorname{Var}\left[\sum_{j=i+1}^{n} \mathrm{P}_{g}\left(x_{j}\right)\right]\right)\right) \\
& +\left(\frac{\Upsilon_{+}^{\prime \prime}\left(\sum_{j=i}^{n} \mathrm{E}\left[\mathrm{P}_{f}\left(x_{j}\right)\right]\right)}{\Upsilon_{+}^{\prime}\left(\sum_{j=i}^{n} \mathrm{E}\left[\mathrm{P}_{f}\left(x_{j}\right)\right]\right)}-\frac{\Upsilon_{+}^{\prime \prime}\left(\sum_{j=i+1}^{n} \mathrm{E}\left[\mathrm{P}_{f}\left(x_{j}\right)\right]\right)}{\Upsilon_{+}^{\prime}\left(\sum_{j=i+1}^{n} \mathrm{E}\left[\mathrm{P}_{f}\left(x_{j}\right)\right]\right)}\right) \\
& \left.\times\left(\operatorname{Var}\left[\sum_{j=i+1}^{n} \mathrm{P}_{f}\left(x_{j}\right)\right]-\operatorname{Var}\left[\sum_{j=i+1}^{n} \mathrm{P}_{g}\left(x_{j}\right)\right]\right)\right\} U_{+}\left(x_{i}\right) \\
& +\sum_{i=1}^{k}\left\{\frac { \hat { \Upsilon } _ { - } ^ { \prime \prime } ( \sum _ { j = 1 } ^ { i } \mathrm { E } [ \mathrm { P } _ { f } ( x _ { j } ) ] ) } { \hat { \Upsilon } _ { - } ^ { \prime } ( \sum _ { j = 1 } ^ { i } \mathrm { E } [ \mathrm { P } _ { f } ( x _ { j } ) ] ) } \left(\operatorname{Var}\left[\sum_{j=1}^{i} \mathrm{P}_{f}\left(x_{j}\right)\right]-\operatorname{Var}\left[\sum_{j=1}^{i-1} \mathrm{P}_{f}\left(x_{j}\right)\right]\right.\right. \\
& \left.-\left(\operatorname{Var}\left[\sum_{j=1}^{i} \mathrm{P}_{g}\left(x_{j}\right)\right]-\operatorname{Var}\left[\sum_{j=1}^{i-1} \mathrm{P}_{g}\left(x_{j}\right)\right]\right)\right) \\
& +\left(\frac{\hat{\Upsilon}_{-}^{\prime \prime}\left(\sum_{j=1}^{i} \mathrm{E}\left[\mathrm{P}_{f}\left(x_{j}\right)\right]\right)}{\hat{\Upsilon}_{-}^{\prime}\left(\sum_{j=1}^{i} \mathrm{E}\left[\mathrm{P}_{f}\left(x_{j}\right)\right]\right)}-\frac{\hat{\Upsilon}_{-}^{\prime \prime}\left(\sum_{j=1}^{i-1} \mathrm{E}\left[\mathrm{P}_{f}\left(x_{j}\right)\right]\right)}{\hat{\Upsilon}_{-}^{\prime}\left(\sum_{j=1}^{i-1} \mathrm{E}\left[\mathrm{P}_{f}\left(x_{j}\right)\right]\right)}\right) \\
& \left.\times\left(\operatorname{Var}\left[\sum_{j=1}^{i-1} \mathrm{P}_{f}\left(x_{j}\right)\right]-\operatorname{Var}\left[\sum_{j=1}^{i-1} \mathrm{P}_{g}\left(x_{j}\right)\right]\right)\right\} U_{-}\left(x_{i}\right) \\
& +o\left(\operatorname{Var}\left[\sum_{i=1}^{n} \mathrm{P}_{f}\left(x_{i}\right)\right]\right)+o\left(\operatorname{Var}\left[\sum_{i=1}^{n} \mathrm{P}_{g}\left(x_{i}\right)\right]\right) .
\end{aligned}
$$

Hence, if the probabilities of each consequence $x \in X$ are mutually independent, 


$$
\begin{aligned}
& 2(V(f)-V(g)) \\
& =\sum_{i=k+1}^{n}\left\{\frac{\Upsilon_{+}^{\prime \prime}\left(\sum_{j=i}^{n} \mathrm{E}\left[\mathrm{P}_{f}\left(x_{j}\right)\right]\right)}{\Upsilon_{+}^{\prime}\left(\sum_{j=i}^{n} \mathrm{E}\left[\mathrm{P}_{f}\left(x_{j}\right)\right]\right)}\left(\operatorname{Var}\left[\mathrm{P}_{f}\left(x_{i}\right)\right]-\operatorname{Var}\left[\mathrm{P}_{g}\left(x_{i}\right)\right]\right)\right. \\
& +\left(\frac{\Upsilon_{+}^{\prime \prime}\left(\sum_{j=i}^{n} \mathrm{E}\left[\mathrm{P}_{f}\left(x_{j}\right)\right]\right)}{\Upsilon_{+}^{\prime}\left(\sum_{j=i}^{n} \mathrm{E}\left[\mathrm{P}_{f}\left(x_{j}\right)\right]\right)}-\frac{\Upsilon_{+}^{\prime \prime}\left(\sum_{j=i+1}^{n} \mathrm{E}\left[\mathrm{P}_{f}\left(x_{j}\right)\right]\right)}{\Upsilon_{+}^{\prime}\left(\sum_{j=i+1}^{n} \mathrm{E}\left[\mathrm{P}_{f}\left(x_{j}\right)\right]\right)}\right) \\
& \left.\times\left(\sum_{j=i+1}^{n}\left(\operatorname{Var}\left[\mathrm{P}_{f}\left(x_{j}\right)\right]-\operatorname{Var}\left[\mathrm{P}_{g}\left(x_{i}\right)\right]\right)\right)\right\} U_{+}\left(x_{i}\right) \\
& +\sum_{i=1}^{k}\left(\frac{\hat{\Upsilon}_{-}^{\prime \prime}\left(\sum_{j=1}^{i} \mathrm{E}\left[\mathrm{P}_{f}\left(x_{j}\right)\right]\right)}{\hat{\Upsilon}_{-}^{\prime}\left(\sum_{j=1}^{i} \mathrm{E}\left[\mathrm{P}_{f}\left(x_{j}\right)\right]\right)}\left(\operatorname{Var}\left[\mathrm{P}_{f}\left(x_{j}\right)\right]-\operatorname{Var}\left[\mathrm{P}_{g}\left(x_{i}\right)\right]\right)\right. \\
& +\left(\frac{\hat{\Upsilon}_{-}^{\prime \prime}\left(\sum_{j=1}^{i} \mathrm{E}\left[\mathrm{P}_{f}\left(x_{j}\right)\right]\right)}{\hat{\Upsilon}_{-}^{\prime}\left(\sum_{j=1}^{i} \mathrm{E}\left[\mathrm{P}_{f}\left(x_{j}\right)\right]\right)}-\frac{\hat{\Upsilon}_{-}^{\prime \prime}\left(\sum_{j=1}^{i-1} \mathrm{E}\left[\mathrm{P}_{f}\left(x_{j}\right)\right]\right)}{\hat{\Upsilon}_{-}^{\prime}\left(\sum_{j=1}^{i-1} \mathrm{E}\left[\mathrm{P}_{f}\left(x_{j}\right)\right]\right)}\right) \\
& \left.\times\left(\sum_{j=1}^{i-1}\left(\operatorname{Var}\left[\mathrm{P}_{f}\left(x_{j}\right)\right]-\operatorname{Var}\left[\mathrm{P}_{g}\left(x_{j}\right)\right]\right)\right)\right) U_{-}\left(x_{i}\right) \\
& +o\left(\sum_{i=1}^{n}\left(\operatorname{Var}\left[\mathrm{P}_{f}\left(x_{i}\right)\right]+\operatorname{Var}\left[\mathrm{P}_{g}\left(x_{i}\right)\right]\right)\right) .
\end{aligned}
$$

From this equation, we obtain the result immediately under the assumptions of Theorem 2.

Theorem 2 shows that, given two acts that are identical except in the degree of ambiguity, any DM who is ambiguity averse (loving) for gain and ambiguity loving (averse) for loss prefers the act with the lower (higher) variance for each positive outcome or gain and the higher (lower) variance for each negative outcome or loss under her/his first-order probabilities to the act with the higher (lower) variance for gain and the lower (higher) variance for loss. Hence, this theorem shows that the measure of ambiguity is given by the variance of the first-order beliefs when ambiguity is represented by the set of first-order probabilities. The accuracy of this measure of ambiguity is determined by the accuracy of the expansion in Theorem 1, which is of order $o\left(\sum_{j=1}^{n}\left(\operatorname{Var}\left[\mathrm{P}_{f}\left(x_{j}\right)\right]+\operatorname{Var}\left[\mathrm{P}_{g}\left(x_{j}\right)\right]\right)\right) \cdot$ [2] proposes a different ambiguity measure defined by

$$
\mho^{2}[f]=\sum_{j=1}^{n} \mathrm{E}\left[\mathrm{P}_{f}\left(x_{j}\right)\right] \operatorname{Var}\left[\mathrm{P}_{f}\left(x_{j}\right)\right] .
$$

However, he considers only a case in which a DM's attitude is symmetrical in loss and gain, that is, he/she is ambiguity averse (or loving) in entire states, regardless of whether the outcome is loss or gain. Hence, $\mho^{2}[f]$ cannot distinguish ambiguity against loss and ambiguity for gain. On the other hand, our ambiguity measure provided by Theorem 2 can be understood more intuitively and it can distinguish ambiguity amounts against loss and for gain.

For each outcome $x_{i}, i=1, \cdots, n$, let $\Delta F_{f+}\left(x_{i}\right)$ and $\Delta \hat{F}_{f-}\left(x_{i}\right)$ be defined 
by

$$
\begin{aligned}
& \Delta F_{f+}\left(x_{i}\right)=\left(\Upsilon_{+}^{-1}\left(\mathrm{E}\left[\Upsilon_{+}\left(\sum_{j=i}^{n} \mathrm{P}_{f}\left(x_{j}\right)\right)\right]\right)-\Upsilon_{+}^{-1}\left(\mathrm{E}\left[\Upsilon_{+}\left(\sum_{j=i+1}^{n} \mathrm{P}_{f}\left(x_{j}\right)\right)\right]\right)\right) \\
& \text { for } i=k+1, \cdots, n, \\
& \text { and } \Delta \hat{F}_{f-}\left(x_{i}\right)=\left(\hat{\Upsilon}_{-}^{-1}\left(\mathrm{E}\left[\hat{\Upsilon}_{-}\left(\sum_{j=1}^{i} \mathrm{P}_{f}\left(x_{j}\right)\right)\right]\right)-\hat{\Upsilon}_{-}^{-1}\left(\mathrm{E}\left[\hat{\Upsilon}_{-}\left(\sum_{j=1}^{i-1} \mathrm{P}_{f}\left(x_{j}\right)\right)\right]\right)\right) \\
& \text { for } i=1, \cdots, k .
\end{aligned}
$$

Then, from (2), $f \succsim_{1} g$ is equivalent to

$$
\begin{aligned}
& \sum_{i=1}^{k} U_{-}\left(x_{i}\right) \Delta \hat{F}_{f-}\left(x_{i}\right)+\sum_{i=k+1}^{n} U_{+}\left(x_{i}\right) \Delta F_{f+}\left(x_{i}\right) \\
& \geq \sum_{i=1}^{k} U_{-}\left(x_{i}\right) \Delta \hat{F}_{g-}\left(x_{i}\right)+\sum_{i=k+1}^{n} U_{+}\left(x_{i}\right) \Delta F_{g+}\left(x_{i}\right) .
\end{aligned}
$$

Replacing $f \succsim_{1} g$ by (13), we obtain the following corollary to the previous theorem.

Corollary 1. Under the same assumptions of Theorem 2, for every act $f, g \in \mathcal{F}_{0}$, if the variance satisfies (8), then, ignoring the small order (9),

$$
\begin{array}{ll}
\Delta F_{f_{+}}\left(x_{i}\right) \geq(\leq) \Delta F_{g+}\left(x_{i}\right), & i=k+1, \cdots, n, \\
\Delta \hat{F}_{f_{-}}\left(x_{i}\right) \leq(\geq) \Delta \hat{F}_{g-}\left(x_{i}\right), & i=1, \cdots, k .
\end{array}
$$

Furthermore, if the DM's attitude toward ambiguity is of the CAAA type, then (8) is the necessary and sufficient condition for (14).

Corollary 1 shows that the plus part of the act $f$ dominates that of the act $g$ w.r.t. the capacity $\Upsilon_{+}^{-1}\left(\mathrm{E}\left[\Upsilon_{+}(\cdot)\right]\right)$ and the minus part of the act $f$ is dominated by that of the act $g$ w.r.t. the capacity $\hat{\Upsilon}_{-}^{-1}\left(E\left[\hat{\Upsilon}_{-}(\cdot)\right]\right)$, which corresponds to the first-order stochastic dominance in the usual risk theory without ambiguity.

Next, we define comparative ambiguity attitudes among DMs using the notion of ambiguity aversion in Definition 1 so that DMs can be ordered by their level of aversion to ambiguity. In order to make this comparison among DMs, we assume that DMs share the same beliefs, i.e., they have the same set of prior $\mathcal{P}$ and the same measure $\xi$ over $\mathcal{P}$, and the same utility functions $U_{+}$and $U_{-}$.

Definition 3. Let $\succsim_{A}^{1}$ and $\succsim_{B}^{1}$ be the preferences over the set of primary acts $\mathcal{F}_{0}$ of two DMs, $\mathrm{A}$ and $\mathrm{B}$, who share the same beliefs and utility functions, respectively. For each event $E \in \mathcal{E}$ and event $E^{*} \in \mathcal{E}$ satisfying (7), DM A is at least as ambiguity averse for gain as $\mathrm{DM} \mathrm{B}$ if

$$
\delta_{E} \succsim_{A}^{1} \delta_{E^{*}} \Rightarrow \delta_{E} \succsim_{B}^{1} \delta_{E^{*}},
$$

and DM $\mathrm{A}$ is at least as ambiguity loving for loss as DM B if

$$
-\delta_{E} \precsim_{A}^{1}-\delta_{E^{*}} \Rightarrow-\delta_{E} \precsim_{B}^{1}-\delta_{E^{*}} .
$$

We obtain the following theorem and corollary in exactly the same way in the result with respect to the concept of "at least risk averse" [25]. 
Theorem 3. Suppose that DMs $A$ and B share the same beliefs and utility function, and they have strictly increasing continuous outlook functions $\left(\Upsilon_{A_{+}}, \Upsilon_{A_{-}}\right)$ and $\left(\Upsilon_{B+}, \Upsilon_{B-}\right)$.DM $A$ is at least as ambiguity averse for gain as DM $B$ whenever $\Upsilon_{A+}=h \circ \Upsilon_{B+}$ for some increasing and concave function $h: \Upsilon_{B+}([0,1]) \rightarrow \mathbb{R}$. $D M A$ is at least as ambiguity loving for loss as DM B whenever $\hat{\Upsilon}_{A-}=\hat{h}_{\circ} \hat{\Upsilon}_{B-}$ for some increasing and concave function $\hat{h}: \hat{\Upsilon}_{B-}([0,1]) \rightarrow \mathbb{R}$.

Applying the Arrow-Pratt risk theory to beliefs, we immediately have the following corollary of Theorem 3.

Corollary 2. Suppose that DMs $A$ and B share the same beliefs and utility. If $\left(\Upsilon_{A+}, \Upsilon_{A-}\right)$ and $\left(\Upsilon_{B+}, \Upsilon_{B-}\right)$ are twice continuously differentiable, then $A$ is at least as ambiguity averse for gain as $B$ whenever

$$
-\frac{\Upsilon_{A+}^{\prime \prime}}{\Upsilon_{A+}^{\prime}} \geq-\frac{\Upsilon_{B+}^{\prime \prime}}{\Upsilon_{B+}^{\prime}} \quad \forall x \in[0,1],
$$

and $\mathrm{A}$ is at least as ambiguity loving for loss as B whenever

$$
-\frac{\hat{\Upsilon}_{A-}^{\prime \prime}}{\hat{\Upsilon}_{A-}^{\prime}} \geq-\frac{\hat{\Upsilon}_{B-}^{\prime \prime}}{\hat{\Upsilon}_{B-}^{\prime}} \quad \forall x \in[0,1] .
$$

\section{Application to the Standard Portfolio Problem}

Consider a DM with an initial wealth $w_{0}$ that he can invest in a risk-free asset and in an ambiguous asset. The return of the risk-free asset is constant $r$. The return of the ambiguous asset over the period is a random $\tilde{x}_{0}$. The ambiguous asset is ambiguous in that the probability distribution of $\tilde{x}_{0}$ is not uniquely given but given by a set $\mathcal{P}$. The problem of the DM is to determine the optimal composition $\left(w_{0}-\alpha, \alpha\right)$ of his portfolio, where $w_{0}-\alpha$ is invested in the risk-free asset and $\alpha$ is invested in the ambiguous asset. The value of the portfolio at the end of the period is written as

$$
\begin{aligned}
& \left(w_{0}-\alpha\right)(1+r)+\alpha\left(1+\tilde{x}_{0}\right) \\
& =w+\alpha \tilde{x},
\end{aligned}
$$

where we use $w=w_{0}(1+r)$ and $\tilde{x}=\tilde{x}_{0}-r$ for notational simplicity. The DM evaluates the value of the portfolio according to EUUP and selects the portfolio in order to maximize its value. In the sequel, we assume $U_{-}(w)=U_{+}(w)=0$ without loss of generality, and only consider the case that $\alpha$ is positive in order to simplify the arguments. Then, denoting $\tilde{x}=\sum_{i=1}^{n} x_{i} \delta_{E_{i}}$, the problem of the $\mathrm{DM}$ is given by

$$
\max _{\alpha} V(\alpha)=\sum_{i=1}^{k} U_{-}\left(w+\alpha x_{i}\right) \Delta \hat{F}_{-}\left(x_{i}\right)+\sum_{i=k+1}^{n} U_{+}\left(w+\alpha x_{i}\right) \Delta F_{+}\left(x_{i}\right),
$$

where

$$
\begin{aligned}
& \Delta F_{+}\left(x_{i}\right)=\left(\Upsilon_{+}^{-1}\left(\mathrm{E}\left[\Upsilon_{+}\left(\sum_{j=i}^{n} \mathrm{P}\left(x_{j}\right)\right)\right]\right)-\Upsilon_{+}^{-1}\left(\mathrm{E}\left[\Upsilon_{+}\left(\sum_{j=i+1}^{n} \mathrm{P}\left(x_{j}\right)\right)\right]\right)\right) \\
& \text { for } i=k+1, \cdots, n,
\end{aligned}
$$




$$
\begin{aligned}
& \Delta \hat{F}_{-}\left(x_{i}\right)=\left(\hat{\Upsilon}_{-}^{-1}\left(\mathrm{E}\left[\hat{\Upsilon}_{-}\left(\sum_{j=1}^{i} \mathrm{P}\left(x_{j}\right)\right)\right]\right)-\hat{\Upsilon}_{-}^{-1}\left(\mathrm{E}\left[\hat{\Upsilon}_{-}\left(\sum_{j=1}^{i-1} \mathrm{P}\left(x_{j}\right)\right)\right]\right)\right) \\
& \text { for } i=1, \cdots, k
\end{aligned}
$$

and where $\mathrm{P}\left(x_{i}\right)=\mathrm{P}\left(\left\{s \in \mathcal{S} \mid \tilde{x}=x_{i}\right\}\right), i=1, \cdots, n$. To hedge the technical difficulty, we assume $V(\alpha)$ is strictly concave w.r.t. $\alpha$ and $\operatorname{argmax} V(\alpha)$ is positive in the sequel.

\subsection{The Impact of Ambiguity Aversion}

Under which condition does a change in attitudes toward ambiguity reduce the investment in the ambiguous asset? To answer this question, let us consider a change of the outlook functions $\left(\Upsilon_{2+}, \Upsilon_{2-}\right)$ to $\left(\Upsilon_{1+}, \Upsilon_{1-}\right)$. By the concavity of the objective function $V$ w.r.t. to $\alpha$, this change reduces the optimal investment in the ambiguous asset if and only if

$$
\begin{gathered}
\sum_{i=1}^{k} x_{i} U_{-}^{\prime}\left(w+\alpha x_{i}\right) \Delta \hat{F}_{2-}\left(x_{i}\right)+\sum_{i=k+1}^{n} x_{i} U_{+}^{\prime}\left(w+\alpha x_{i}\right) \Delta F_{2+}\left(x_{i}\right)=0 \\
\Rightarrow \sum_{i=1}^{k} x_{i} U_{-}^{\prime}\left(w+\alpha x_{i}\right) \Delta \hat{F}_{1-}\left(x_{i}\right)+\sum_{i=k+1}^{n} x_{i} U_{+}^{\prime}\left(w+\alpha x_{i}\right) \Delta F_{1+}\left(x_{i}\right) \leq 0,
\end{gathered}
$$

where $\Delta F_{i+}$ and $\Delta \hat{F}_{i-}, i=1,2$, are $\Delta F_{+}$and $\Delta \hat{F}_{-}$in which $\left(\Upsilon_{+}, \Upsilon_{-}\right)$is replaced by $\left(\Upsilon_{i+}, \Upsilon_{i-}\right), i=1,2$, respectively.

Proposition 4. Consider a change in attitude toward ambiguity from $\left(\Upsilon_{2+}, \Upsilon_{2-}\right)$ to $\left(\Upsilon_{1+}, \Upsilon_{1-}\right)$. Suppose the assumptions of Theorem 2 hold. Then, this change reduces the optimal investment in the ambiguous asset if

a) $\left(\Upsilon_{1+}, \Upsilon_{1-}\right)$ is at least as ambiguity averse for both gain and loss as $\left(\Upsilon_{2+}, \Upsilon_{2-}\right)$,

b) the slope of the absolute ambiguity aversion of $\Upsilon_{1+}$ is steeper than that of $\Upsilon_{2+}$ and that of $\hat{\Upsilon}_{2-}$ is steeper than that of $\hat{\Upsilon}_{1-}$;

$$
\left(\frac{\Upsilon_{1+}^{\prime \prime}(x)}{\Upsilon_{1+}^{\prime}(x)}\right)^{\prime} \leq\left(\frac{\Upsilon_{2+}^{\prime \prime}(x)}{\Upsilon_{2+}^{\prime}(x)}\right)^{\prime} \leq 0 \text { and }\left(\frac{\hat{\Upsilon}_{1-}^{\prime \prime}(x)}{\hat{\Upsilon}_{1-}^{\prime}(x)}\right)^{\prime} \geq\left(\frac{\hat{\Upsilon}_{2-}^{\prime \prime}(x)}{\hat{\Upsilon}_{2-}^{\prime}(x)}\right)^{\prime} \geq 0
$$

c) and the relative risk aversions $x U_{+}^{\prime \prime}(x) / U_{+}^{\prime}(x)$ and $x U_{-}^{\prime \prime}(x) / U_{-}^{\prime}(x)$ are less than unity.

Proof. Let $\phi_{+}(x)=x U_{+}^{\prime}\left(w_{0}+\alpha x\right)$ and $\phi_{-}(x)=x U_{-}^{\prime}\left(w_{0}+\alpha x\right)$. Then, the sufficient condition of the reduction of the optimal investment in the ambiguous asset is given by

$$
\begin{aligned}
& \sum_{i=1}^{k} \phi_{-}\left(x_{i}\right) \Delta \hat{F}_{1-}\left(x_{i}\right)+\sum_{i=k+1}^{n} \phi_{+}\left(x_{i}\right) \Delta F_{1+}\left(x_{i}\right) \\
& \leq \sum_{i=1}^{k} \phi_{-}\left(x_{i}\right) \Delta \hat{F}_{2-}\left(x_{j}\right)+\sum_{i=k+1}^{n} \phi_{+}\left(x_{i}\right) \Delta F_{2+}\left(x_{i}\right),
\end{aligned}
$$

or

$$
\sum_{i=1}^{k} \phi_{-}\left(x_{i}\right)\left(\Delta \hat{F}_{1-}\left(x_{i}\right)-\Delta \hat{F}_{2-}\left(x_{i}\right)\right)+\sum_{i=k+1}^{n} \phi_{+}\left(x_{i}\right)\left(\Delta F_{1+}\left(x_{i}\right)-\Delta F_{2+}\left(x_{i}\right)\right) \leq 0 \text {. }
$$


We can easily show that $\phi_{+}$and $\phi_{-}$are increasing if the condition (c) is satisfied (see Proposition 4.9 of [26]). Hence, we have

$$
\phi_{-}(x)<0 \text { for negative } x \in X \text {, and } \phi_{+}(x)>0 \text { for positive } x \in X \text {. }
$$

On the other hand, by the same arguments as the derivation of (12), we have

$$
\begin{aligned}
& 2\left(\Delta F_{1+}\left(x_{i}\right)-\Delta F_{2+}\left(x_{i}\right)\right) \\
& =\left(\frac{\Upsilon_{1+}^{\prime \prime}\left(\sum_{j=i}^{n} \mathrm{E}\left[\mathrm{P}\left(x_{j}\right)\right]\right)}{\Upsilon_{1+}^{\prime}\left(\sum_{j=i}^{n} \mathrm{E}\left[\mathrm{P}\left(x_{j}\right)\right]\right)}-\frac{\Upsilon_{2+}^{\prime \prime}\left(\sum_{j=i}^{n} \mathrm{E}\left[\mathrm{P}\left(x_{j}\right)\right]\right)}{\Upsilon_{2+}^{\prime}\left(\sum_{j=i}^{n} \mathrm{E}\left[\mathrm{P}\left(x_{j}\right)\right]\right)}\right) \operatorname{Var}\left[\mathrm{P}\left(x_{i}\right)\right] \\
& +\left(\left(\frac{\Upsilon_{1+}^{\prime \prime}\left(\sum_{j=i}^{n} \mathrm{E}\left[\mathrm{P}\left(x_{j}\right)\right]\right)}{\Upsilon_{1+}^{\prime}\left(\sum_{j=i}^{n} \mathrm{E}\left[\mathrm{P}\left(x_{j}\right)\right]\right)}-\frac{\Upsilon_{1+}^{\prime \prime}\left(\sum_{j=i+1}^{n} \mathrm{E}\left[\mathrm{P}\left(x_{j}\right)\right]\right)}{\Upsilon_{1+}^{\prime}\left(\sum_{j=i+1}^{n} \mathrm{E}\left[\mathrm{P}\left(x_{j}\right)\right]\right)}\right)\right. \\
& \left.-\left(\frac{\Upsilon_{2+}^{\prime \prime}\left(\sum_{j=i}^{n} \mathrm{E}\left[\mathrm{P}\left(x_{j}\right)\right]\right)}{\Upsilon_{2+}^{\prime}\left(\sum_{j=i}^{n} \mathrm{E}\left[\mathrm{P}\left(x_{j}\right)\right]\right)}-\frac{\Upsilon_{2+}^{\prime \prime}\left(\sum_{j=i+1}^{n} \mathrm{E}\left[\mathrm{P}\left(x_{j}\right)\right]\right)}{\Upsilon_{2+}^{\prime}\left(\sum_{j=i+1}^{n} \mathrm{E}\left[\mathrm{P}\left(x_{j}\right)\right]\right)}\right)\right) \sum_{j=i+1}^{n} \operatorname{Var}\left[\mathrm{P}\left(x_{j}\right)\right] \\
& +o\left(\sum_{i=1}^{n} \operatorname{Var}\left[\mathrm{P}\left(x_{i}\right)\right]\right) \quad \text { for } i=k+1, \cdots, n \text {, } \\
& 2\left(\Delta \hat{F}_{1-}\left(x_{i}\right)-\Delta \hat{F}_{2-}\left(x_{i}\right)\right) \\
& =\left(\frac{\hat{\Upsilon}_{1-}^{\prime \prime}\left(\sum_{j=1}^{i} \mathrm{E}\left[\mathrm{P}\left(x_{j}\right)\right]\right)}{\hat{\Upsilon}_{1-}^{\prime}\left(\sum_{j=1}^{i} \mathrm{E}\left[\mathrm{P}\left(x_{j}\right)\right]\right)}-\frac{\hat{\Upsilon}_{2-}^{\prime \prime}\left(\sum_{j=1}^{i} \mathrm{E}\left[\mathrm{P}\left(x_{j}\right)\right]\right)}{\hat{\Upsilon}_{2-}^{\prime}\left(\sum_{j=1}^{i} \mathrm{E}\left[\mathrm{P}\left(x_{j}\right)\right]\right)}\right) \operatorname{Var}\left[\mathrm{P}\left(x_{i}\right)\right] \\
& +\left(\left(\frac{\hat{\Upsilon}_{1-}^{\prime \prime}\left(\sum_{j=1}^{i} \mathrm{E}\left[\mathrm{P}\left(x_{j}\right)\right]\right)}{\hat{\Upsilon}_{1-}^{\prime}\left(\sum_{j=1}^{i} \mathrm{E}\left[\mathrm{P}\left(x_{j}\right)\right]\right)}-\frac{\hat{\Upsilon}_{1-}^{\prime \prime}\left(\sum_{j=1}^{i-1} \mathrm{E}\left[\mathrm{P}\left(x_{j}\right)\right]\right)}{\hat{\Upsilon}_{1-}^{\prime}\left(\sum_{j=1}^{i-1} \mathrm{E}\left[\mathrm{P}\left(x_{j}\right)\right]\right)}\right)\right. \\
& -\left(\frac{\hat{\Upsilon}_{2-}^{\prime \prime}\left(\sum_{j=1}^{i} \mathrm{E}\left[\mathrm{P}\left(x_{j}\right)\right]\right)}{\hat{\Upsilon}_{2-}^{\prime}\left(\sum_{j=1}^{i} \mathrm{E}\left[\mathrm{P}\left(x_{j}\right)\right]\right)}-\frac{\hat{\Upsilon}_{2-}^{\prime \prime}\left(\sum_{j=1}^{i-1} \mathrm{E}\left[\mathrm{P}\left(x_{j}\right)\right]\right)}{\hat{\Upsilon}_{2-}^{\prime}\left(\sum_{j=1}^{i-1} \mathrm{E}\left[\mathrm{P}\left(x_{j}\right)\right]\right)}\right) \sum_{j=1}^{i-1} \operatorname{Var}\left[\mathrm{P}\left(x_{j}\right)\right] \\
& +o\left(\sum_{i=1}^{n} \operatorname{Var}\left[\mathrm{P}\left(x_{i}\right)\right]\right) \quad \text { for } i=1, \cdots, k \text {. }
\end{aligned}
$$

Noting that the condition (a) is equivalent to $\frac{\Upsilon_{1+}^{\prime \prime}(x)}{\Upsilon_{1+}^{\prime}(x)} \leq \frac{\Upsilon_{2+}^{\prime \prime}(x)}{\Upsilon_{2+}^{\prime}(x)}$ and $\frac{\hat{\Upsilon}_{1-}^{\prime \prime}(x)}{\hat{\Upsilon}_{1-}^{\prime}(x)} \geq \frac{\hat{\Upsilon}_{2-}^{\prime \prime}(x)}{\hat{\Upsilon}_{2-}^{\prime}(x)}$ from Corollary 2, the conditions (a) and (b) imply

$$
\begin{gathered}
\Delta \hat{F}_{1-}\left(x_{i}\right)-\Delta \hat{F}_{2-}\left(x_{i}\right) \geq 0, i=1, \cdots, k, \\
\Delta F_{1+}\left(x_{i}\right)-\Delta F_{2+}\left(x_{i}\right) \leq 0, i=k+1, \cdots, n .
\end{gathered}
$$

Combining (17) and (20), we obtain the result.

\subsection{The Impact of a Change in Ambiguity}

Finally, we consider a question: under which condition does a change in ambiguity of return increase the demand for the ambiguous asset? Let $\tilde{x}_{1}$ and $\tilde{x}_{2}$ 
be excess returns of the ambiguous asset, and let $P_{1}$ and $P_{2}$ be probability distributions under the first-order probabilities defined by

$$
\begin{gathered}
\mathrm{P}_{1}\left(x_{i}\right)=\mathrm{P}\left(s \in S \mid \tilde{x}_{1}(s)=x_{i}\right), \\
\text { and } \mathrm{P}_{2}\left(x_{i}\right)=\mathrm{P}\left(s \in S \mid \tilde{x}_{2}(s)=x_{i}\right), \quad i=1, \cdots, n \text {, respectively. }
\end{gathered}
$$

In this subsection, we denote $\Delta F_{+}$in (15) and $\Delta \hat{F}_{-}$in (16) as $\Delta F_{i+}$ and $\Delta \hat{F}_{i-}$, respectively, $i=1,2$, in the case where $\mathrm{P}$ is replaced by $\mathrm{P}_{i}$. The problem is to determine the condition under which we have

$$
\begin{aligned}
& \sum_{i=1}^{k} x_{i} U_{-}^{\prime}\left(w+\alpha x_{i}\right) \Delta \hat{F}_{1-}\left(x_{i}\right)+\sum_{i=k+1}^{n} x_{i} U_{+}^{\prime}\left(w+\alpha x_{i}\right) \Delta F_{1+}\left(x_{i}\right)=0 \\
\Rightarrow & \sum_{i=1}^{k} x_{i} U_{-}^{\prime}\left(w+\alpha x_{i}\right) \Delta \hat{F}_{2-}\left(x_{i}\right)+\sum_{i=k+1}^{n} x_{i} U_{+}^{\prime}\left(w+\alpha x_{i}\right) \Delta F_{2+}\left(x_{i}\right) \geq 0 .
\end{aligned}
$$

or

$$
\begin{aligned}
& \sum_{i=1}^{k} x_{i} U_{-}^{\prime}\left(w+\alpha x_{i}\right)\left(\Delta \hat{F}_{1-}\left(x_{i}\right)-\Delta \hat{F}_{2-}\left(x_{i}\right)\right) \\
& +\sum_{i=k+1}^{n} x_{i} U_{+}^{\prime}\left(w+\alpha x_{i}\right)\left(\Delta F_{1+}\left(x_{i}\right)-\Delta F_{2+}\left(x_{i}\right)\right) \leq 0 .
\end{aligned}
$$

Proposition 5. Consider a change in ambiguity of the ambiguous asset return from $\tilde{x}_{2}$ to $\tilde{x}_{1}$. Suppose the assumptions of Theorem 2 hold. Then, this change reduces (increases) the optimal investment in the ambiguous asset if

a) a DM is ambiguity averse (loving) for gain and ambiguity loving (averse) for loss,

b) both $-\frac{\Upsilon_{+}^{\prime \prime}}{\Upsilon_{+}^{\prime}}$ and $-\frac{\hat{\Upsilon}_{-}^{\prime \prime}}{\hat{\Upsilon}_{-}^{\prime}}$ are increasing (decreasing),

c) $\tilde{x}_{1}$ is more ambiguous than $\tilde{x}_{2}$ for gain and $\tilde{x}_{1}$ is less ambiguous than $\tilde{x}_{2}$ for loss;

$$
\begin{gathered}
\mathrm{E}\left[\mathrm{P}_{1}\left(x_{i}\right)\right]=\mathrm{E}\left[\mathrm{P}_{2}\left(x_{i}\right)\right], i=1, \cdots, n, \\
\begin{cases}\operatorname{Var}\left[\mathrm{P}_{1}\left(x_{i}\right)\right] \leq \operatorname{Var}\left[\mathrm{P}_{2}\left(x_{i}\right)\right], \quad i=1, \cdots, k, \\
\operatorname{Var}\left[\mathrm{P}_{1}\left(x_{i}\right)\right] \geq \operatorname{Var}\left[\mathrm{P}_{2}\left(x_{i}\right)\right], \quad i=k+1, \cdots, n,\end{cases}
\end{gathered}
$$

d) and relative risk aversions $x U_{+}^{\prime \prime}(x) / U_{+}^{\prime}(x)$ and $x U_{-}^{\prime \prime}(x) / U_{-}^{\prime}(x)$ are less than unity.

Proof. In the same way as in (18), we have

$$
\begin{aligned}
2 & \left(\Delta F_{1+}\left(x_{i}\right)-\Delta F_{2+}\left(x_{i}\right)\right) \\
= & \frac{\Upsilon_{+}^{\prime \prime}\left(\sum_{j=i}^{n} \mathrm{E}\left[\mathrm{P}_{1}\left(x_{j}\right)\right]\right)}{\Upsilon_{+}^{\prime}\left(\sum_{j=i}^{n} \mathrm{E}\left[\mathrm{P}_{1}\left(x_{j}\right)\right]\right)}\left(\operatorname{Var}\left[\mathrm{P}_{1}\left(x_{i}\right)\right]-\operatorname{Var}\left[\mathrm{P}_{2}\left(x_{i}\right)\right]\right) \\
& +\left(\frac{\Upsilon_{+}^{\prime \prime}\left(\sum_{j=i}^{n} \mathrm{E}\left[\mathrm{P}_{1}\left(x_{j}\right)\right]\right)}{\Upsilon_{+}^{\prime}\left(\sum_{j=i}^{n} \mathrm{E}\left[\mathrm{P}_{1}\left(x_{j}\right)\right]\right)}-\frac{\Upsilon_{+}^{\prime \prime}\left(\sum_{j=i+1}^{n} \mathrm{E}\left[\mathrm{P}_{1}\left(x_{j}\right)\right]\right)}{\Upsilon_{+}^{\prime}\left(\sum_{j=i+1}^{n} \mathrm{E}\left[\mathrm{P}_{1}\left(x_{j}\right)\right]\right)}\right) \sum_{j=i+1}^{n}\left(\operatorname{Var}\left[\mathrm{P}_{1}\left(x_{j}\right)\right]\right. \\
& \left.-\operatorname{Var}\left[\mathrm{P}_{2}\left(x_{j}\right)\right]\right)+o\left(\sum_{i=1}^{n}\left(\operatorname{Var}\left[\mathrm{P}_{1}\left(x_{i}\right)\right]+\operatorname{Var}\left[\mathrm{P}_{2}\left(x_{i}\right)\right]\right)\right) \text { for } i=k+1, \cdots, n,
\end{aligned}
$$




$$
\begin{aligned}
& 2\left(\Delta \hat{F}_{1-}\left(x_{i}\right)-\Delta \hat{F}_{2-}\left(x_{i}\right)\right) \\
& =\frac{\hat{\Upsilon}_{-}^{\prime \prime}\left(\sum_{j=1}^{i} \mathrm{E}\left[\mathrm{P}_{1}\left(x_{j}\right)\right]\right)}{\hat{\Upsilon}_{-}^{\prime}\left(\sum_{j=1}^{i} \mathrm{E}\left[\mathrm{P}_{1}\left(x_{j}\right)\right]\right)}\left(\operatorname{Var}\left[\mathrm{P}_{1}\left(x_{i}\right)\right]-\operatorname{Var}\left[\mathrm{P}_{2}\left(x_{i}\right)\right]\right) \\
& +\left(\frac{\hat{\Upsilon}_{-}^{\prime \prime}\left(\sum_{j=1}^{i} \mathrm{E}\left[\mathrm{P}_{1}\left(x_{j}\right)\right]\right)}{\hat{\Upsilon}_{-}^{\prime}\left(\sum_{j=1}^{i} \mathrm{E}\left[\mathrm{P}_{1}\left(x_{j}\right)\right]\right)}-\frac{\hat{\Upsilon}_{-}^{\prime \prime}\left(\sum_{j=1}^{i-1} \mathrm{E}\left[\mathrm{P}_{1}\left(x_{j}\right)\right]\right)}{\hat{\Upsilon}_{-}^{\prime}\left(\sum_{j=1}^{i-1} \mathrm{E}\left[\mathrm{P}_{1}\left(x_{j}\right)\right]\right)} \sum_{j=1}^{i-1}\left(\operatorname{Var}\left[\mathrm{P}_{1}\left(x_{j}\right)\right]\right.\right. \\
& \left.-\operatorname{Var}\left[\mathrm{P}_{2}\left(x_{j}\right)\right]\right)+o\left(\sum_{i=1}^{n}\left(\operatorname{Var}\left[\mathrm{P}_{1}\left(x_{i}\right)\right]+\operatorname{Var}\left[\mathrm{P}_{2}\left(x_{i}\right)\right]\right)\right) \quad \text { for } i=1, \cdots, k .
\end{aligned}
$$

Hence, by the similar arguments to the proof of Proposition 4, we obtain the result.

\section{Concluding Remarks}

This paper derives an ambiguity measure under expected utility with uncertain probability (EUUP) by [1]. We show that the degree of ambiguity can be measured by the variance of uncertain probabilities just as the degree of risk can be measured by the variance of outcomes. Although [2] also derives an ambiguity measure based on the variance of uncertain probabilities, our measure is more flexible and discriminates between ambiguity in favorable outcomes and in unfavorable ones. We also provide a definition to show that one is at least as ambiguity averse (loving) for gain/loss as another and characterize this by the forms of their outlook functions. Finally, we discuss the effects of ambiguity on financial investments through comparative statics based on our ambiguity measure. We show two sufficient conditions. One is that a change in attitude toward ambiguity reduces the investment in ambiguous assets. The other is that a change in ambiguity of return increases the demand for the ambiguous assets. We derive our ambiguity measure by applying a quadratic approximation to the DMs perceived probabilities. Verification of the accuracy of this approximation is a remaining question. This issue needs to be considered. We will conduct empirical research to validate our ambiguity measure and confirm its usefulness in the near future.

\section{Acknowledgements}

This paper is partly supported by a Grant-in-Aid for Scientific Research (c).

\section{Conflicts of Interest}

The author declares no conflicts of interest regarding the publication of this paper.

\section{References}

[1] Izhakian, Y. (2017) Expected Utility with Uncertain Probability Theory. Journal of Mathematical Economics, 69, 91-103. https://doi.org/10.1016/j.jmateco.2016.12.004

[2] Izhakian, Y. (2020) A Theoretical Foundation of Ambiguity Measurement. Journal 
of Economic Theory, 187, Article ID: 105001.

https://doi.org/10.1016/j.jet.2020.105001

[3] Wakker, P. (2010) Prospect Theory: For Risk and Ambiguity. Cambridge University Press, Cambridge. https://doi.org/10.1017/CBO9780511779329

[4] Knight, F.M. (1921) Risk, Uncertainty and Profit. Houghton Mifflin, Boston.

[5] Schmeidler, D. (1989) Subjective Probability and Expected Utility without Additivity. Econometrica, 57, 571-587. https://doi.org/10.2307/1911053

[6] Gilboa, I. and Schmeidler, D. (1989) Maxmin Expected Utility with Non-Unique Prior. Journal of Mathematical Economics, 18, 141-153. https://doi.org/10.1016/0304-4068(89)90018-9

[7] Tversky, A. and Kahneman, D. (1992) Advances in Prospect Theory: Cumulative Representation of Uncertainty. Journal of Risk and Uncertainty, 5, 297-323. https://doi.org/10.1007/BF00122574

[8] Ghirardato, P., Klibanoff, P. and Marinacci, M. (1998) Additivity with Multiple Priors. Journal of Mathematical Economics, 30, 405-420. https://doi.org/10.1016/S0304-4068(97)00047-5

[9] Klibanoff, P., Marinacci, M. and Mukerji, S. (2005) A Smooth Model of Decision Making under Ambiguity. Econometrica, 73, 1849-1892. https://doi.org/10.1111/j.1468-0262.2005.00640.x

[10] Kocher, M., Lahno, A.M. and Trautmann, S. (2018) Ambiguity Aversion Is Not Universal. European Economic Review, 101, 268-283. https://doi.org/10.1016/j.euroecorev.2017.09.016

[11] Trautmann, S. and Wakker, P. (2018) Making the Anscombe-Aumann Approach to Ambiguity Suitable for Descriptive Applications. Journal of Risk and Uncertainty, 56, 83-116. https://doi.org/10.1007/s11166-018-9273-7

[12] Bossaerts, P., Paolo Ghirardato, P., Guarnaschelli, S. and Zame, W.R. (2010) Ambiguity in Asset Markets: Theory and Experiment. The Review of Financial Studies, 23, 1325-1359. https://doi.org/10.1093/rfs/hhp106

[13] Dow, J. and Werlang, S.R.d.C. (1992) Uncertainty Aversion, Risk Aversion, and the Optimal Choice of Portfolio. Econometrica, 60, 197-204. https://doi.org/10.2307/2951685

[14] Baillon, A., Huang, Z., Selim, A. and Wakker, P.P. (2018) Measuring Ambiguity Attitudes for All (Natural) Events. Econometrica, 86, 1839-1858. https://doi.org/10.3982/ECTA14370

[15] Baillon, A. and Bleichrodt, H. (2015) Testing Ambiguity Models through the Measurement of Probabilities for Gains and Losses. American Economic Journal: Microeconomics, 7, 77-100. https://doi.org/10.1257/mic.20130196

[16] Ui, T. (2011) The Ambiguity Premium vs. the Risk Premium under Limited Market Participation. Review of Finance, 15, 245-275. https://doi.org/10.1093/rof/rfq012

[17] Bewley, T.F. (2011) Knightian Decision Theory and Econometric Inferences. Journal of Economic Theory, 146, 1134-1147. https://doi.org/10.1016/j.jet.2010.12.012

[18] Boyle, P.P., Garlappi, L., Uppal, R. and Wang, T. (2012) Keynes Meets Markowitz: The Trade-Off between Familiarity and Diversification. Management Science, 58, 253-272. https://doi.org/10.1287/mnsc.1110.1349

[19] Nau, R.F. (2006) Uncertainty Aversion with Second-Order Utilities and Probabilities. Management Science, 52, 136-145. https://doi.org/10.1287/mnsc.1050.0469

[20] Chew, S.H. and Sagi, J.S. (2008) Small Worlds: Modeling Attitudes toward Sources 
of Uncertainty. Journal of Economic Theory, 139, 1-24.

https://doi.org/10.1016/j.jet.2007.07.004

[21] Jewitt, I. and Mukerji, S. (2017) Ordering Ambiguous Acts. Journal of Economic Theory, 171, 213-267. https://doi.org/10.1016/j.jet.2017.07.001

[22] Chen, L., Peng, J., Zhang, B. and Rosyida, I. (2017) Diversified Models for Portfolio Selection Based on Uncertain Semivariance. International Journal of Systems Science, 48, 637-648. https://doi.org/10.1080/00207721.2016.1206985

[23] Kopylov, I. (2010) Simple Axioms for Countably Additive Subjective Probability. Journal of Mathematical Economics, 46, 867-876. https://doi.org/10.1016/j.jmateco.2010.07.002

[24] Rothschild, M. and Stiglitz, J.E. (1970) Increasing Risk: I. A Definition. Journal of Economic Theory, 2, 225-243. https://doi.org/10.1016/0022-0531(70)90038-4

[25] Nielsen, L.T. (1988) Comparative Risk Aversion. Economics Letters, 27, 321-325. https://doi.org/10.1016/0165-1765(88)90156-5

[26] Gollier, C. (2001) The Economics of Risk and Time. The MIT Press, Cambridge. https://doi.org/10.7551/mitpress/2622.001.0001 